\title{
Model for Power Consumption of Wireless Access Networks
}

\author{
Margot Deruyck, Emmeric Tanghe, Wout Joseph, Willem Vereecken, \\ Mario Pickavet, Luc Martens, and Bart Dhoedt \\ Ghent University / IBBT, Dept. of Information Technology \\ Gaston Crommenlaan 8 box 201, B-9050 Ghent, Belgium \\ Fax: +32 933 14899, E-mail: margot.deruyck@intec.UGent.be
}

\begin{abstract}
The power consumption of wireless access networks will become an important issue in the coming years. In this paper, the power consumption of base stations for mobile WiMAX, fixed WiMAX, UMTS, HSPA, and LTE is modeled and related to the coverage. A new metric, the power consumption per covered area $P C_{a r e a}$, is introduced, to compare the energy efficiency of the considered technologies for a basic reference configuration and a future extended configuration, which makes use of novel MIMO technology. The introduction of MIMO has a positive influence on the energy efficiency: e.g., for a 4x4 MIMO system, $P C_{\text {area }}$ decreases with $63 \%$ for mobile WiMAX and with $50 \%$ for HSPA and LTE, compared to a SISO system. However, a higher MIMO array size (i.e., a higher number of transmitting and receiving antennas) does not always result in a higher energy efficiency gain.
\end{abstract}

\section{Introduction}

Recent studies have shown that the power consumption of ICT is approximately $4 \%$ of the annual energy production [1]. More importantly, this number is expected to grow drastically in the coming years [1]. Furthermore, the radio access networks are large contributors to the $\mathrm{CO}_{2}$ emissions [1-3]. This indicates that the power consumption of wireless access networks, and more in particular the power consumption of the base stations, is going to become an important issue in the coming years. Nowadays, the base stations are responsible for roughly two-thirds of the total $\mathrm{CO}_{2}$ emissions of the wireless access networks. [3] states that the daily energy consumption per customer is $0.83 \mathrm{Wh}$ for a terminal and $120 \mathrm{Wh}$ for the mobile network which is a consumption ratio of terminal versus network of about 1:150. The energy consumption 
of the terminals is thus negligible in comparison with the energy consumption of the networks. Therefore, it is clear that one should focus on the reduction of energy consumption of base stations in wireless access networks as the terminals are already optimized in terms of energy consumption because they are powered by batteries.

The objective of this paper is to model the power consumption of base stations of various wireless technologies and compare their energy efficiency versus the coverage range. In order to determine the energy efficiency of the considered technologies, a new metric, namely the power consumption per covered area, is defined. The energy efficiency for mobile WiMAX, fixed WiMAX, UMTS, HSPA and LTE is compared for bit rates of 3 and 60 Mbps. Finally, the influence of MIMO(Multiple Input Multiple Output) is investigated.

In literature, some related work can be found. In [4-6], a power consumption model for a base station is proposed. However, in the cited work, it is very difficult to investigate the influence of the individual components of the base station on the total power consumption, as well as the influence of possible dependencies between the components of the base station. Furthermore, in the cited work only one technology is used to determine the power consumption. Our work will show that for the considered case and based on the assumptions made for the parameters, distinct differences in energy efficiency can be noticed between the considered technologies.

The outline of the paper is as follow. In Section 2, a short overview of the considered technologies is given. In Section 3, the power consumption of a base station is modelled and related to the coverage. Section 4 gives some results obtained with the model from Section 3. In Section 5 the final conclusions are given.

\section{Technologies}

For the wireless access networks, we investigate the power consumption of outdoor base stations for five different wireless technologies: mobile WiMAX (Worldwide Interoperability for Microwave Access) [7], fixed WiMAX [8], UMTS (Universal Mobile Telecommunications System) [9], HSPA (High Speed Packet Access) [10] and LTE (Long Term Evolution) [11]. We first give a short description of the different technologies.

WiMAX is a wireless technology for broadband communication based on the IEEE 802.16 standard. For fixed WiMAX, we analyse the IEEE 802.16-2004 interface, operating in the 2-11 GHz band and developed for fixed wireless applications. For mobile WiMAX, we analyse the IEEE 802.16e interface, operating in the 2-6 GHz band and developed for mobile wireless applications. Fixed WiMAX uses OFMDA (Orthogonal Frequency Division Multiple Access) while mobile WiMAX uses the novel SOFDMA (Scalable 
Orthogonal Frequency Division Multiple Access) technique which is derived from OFDMA and supports a wide range of bandwidths to flexibly address the need for various spectrum allocation and application requirements.

UMTS is developed by ETSI (European Telecommunications Standardisation Institute) and operates in the $2.1 \mathrm{GHz}$ band. UMTS has been specified as an integrated solution for mobile voice and data. It offers mobile operators significant capacity and broadband capabilities to support more voice and data consumers, especially in urban centres. UMTS uses W-CDMA (Wideband Code Division Multiple Access) as multiple access technique.

HSPA is the successor of the widely deployed UMTS and works in the $2.1 \mathrm{GHz}$ band. It promises higher data rates, increased cell and user throughput and reduced delay compared to UMTS.

LTE is the newest wireless broadband technology. In December 2009, the world's first publicly available LTE-service was started in Scandinavia [12]. LTE is marketed as the fourth generation (4G) of radio technologies. It uses SOFDMA as multiple access technique and thus supports variable bandwidths from 1.4 to $20 \mathrm{MHz}$, just like mobile WiMAX supports scalability. LTE uses the $2.6 \mathrm{GHz}$ band. In the future, LTE will probably also use the $800 \mathrm{MHz}$ band (digital dividend frequencies).

\section{Theoretical power consumption and coverage model for wire- less access}

\subsection{Power consumption of a base station}

A base station is here defined as the equipment needed to communicate with the mobile stations and with the backhaul network. In a base station, we typically find several power consuming components. Fig. 1 gives an overview of these components [13-15]. The area covered by a base station is called a cell. Each cell is further divided in a number of sectors. Each sector is covered by a sector antenna, which is a directional antenna with a sector-shaped radiation pattern. Some equipment is used for each sector such as the digital signal processing (responsible for system processing and coding), the power amplifier, the transceiver (responsible for receiving and sending of signals to the mobile stations), and the rectifier. The power consumption of these components should be multiplied with the number of supported sectors $n_{\text {sector }}$ when determining the power consumption of the base station. In contrary to $[16,17]$, it is assumed that the signal generator is part of the transceiver. This adaptation is based on the information retrieved from operators. Furthermore, a base station contains equipment that is common for all the sectors such as the air conditioning and the microwave link (responsible for communication with the backhaul network in case no fiber link is available). The distinction between the components per sector and the components 
common for all sectors is based on the information retrieved from operators. In Fig. 1, the equipment of the base station and the different notations for the power consumption $P_{e l}$ of the different components are indicated.

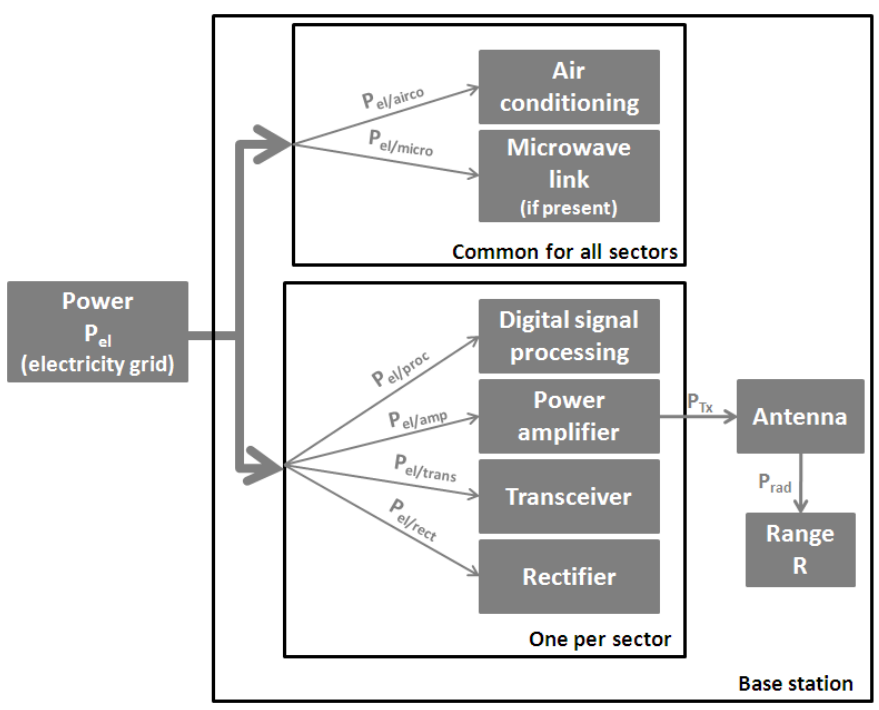

Figure 1: Block diagram of the base station equipment.

The power consumption of each component is here assumed to be constant, except for the power amplifier and the air conditioning. The power consumption of the latter depends on the internal and ambient temperature of the base station cabinet [18]. We assumed an internal and ambient of temperature of $25^{\circ} \mathrm{C}$. To model the power consumption of the power amplifier, the efficiency $\eta$ of the power amplifier is defined which is the ratio of the RF output power $P_{\text {out } / a m p}$ (in Watt) to the electrical input power $P_{\text {el/amp }}$ of the power amplifier (in Watt) [19]. In Fig. 1, $P_{\text {out } / a m p}$ corresponds to the input power $P_{T x}$ of one sector antenna resulting in the following equation for the efficiency $\eta$ :

$$
\eta=\frac{P_{T x}}{P_{e l / a m p}}
$$

Based on $P_{T x}$, we can calculate the power consumption $P_{e l / a m p}$ of the power amplifier (in Watt) as follows:

$$
P_{e l / a m p}=\frac{P_{T x}}{\eta}
$$

Once the power consumption of the different components of the base station is know, the power consumption $P_{e l}$ of the entire base station (in Watt) can be determined:

$$
P_{\text {el }}=n_{\text {sector }} \cdot\left(n_{T x} \cdot\left(P_{\text {el } / a m p}+P_{\text {el/trans }}\right)+P_{\text {el/proc }}+P_{\text {el } / \text { rect }}\right)+P_{\text {el } / \text { micro }}+P_{\text {el/airco }}
$$


with $n_{\text {sector }}$ the number of sectors in the cell, $P_{\text {el/amp }}, P_{\text {el/trans }}, P_{\text {el/proc }}, P_{\text {el/rect }}, P_{\text {el/micro }}$ and $P_{\text {el/airco }}$ are the power consumptions of the power amplifier, the transceiver, the digital signal processing, the rectifier, the microwave link (if present) and the air conditioning, respectively. In case MIMO is used, the base station needs the same number of power amplifiers and the same number of transceivers as the number of transmitting antennas [20]. In order to take the power consumption of this extra equipment into account, the power consumption of the power amplifier and the transceiver is multiplied by the number $n_{T x}$ of transmitting antennas for one sector. MIMO has also an influence on the digital signal processing which is, compared to the transceiver, negligible. Furthermore, eq. (3) is only valid when one frequency is used per sector.

Table 1 summarises the power consumption of the different components of a base station for the considered technologies. These values are retrieved from data sheets of various manufacturers of network equipment and from standards [13,21-28]. For the power amplifier, the maximum power consumption is indicated. The power consumption of the digital signal processing and the transceiver are based on confidential data retrieved from an operator. The results presented in this paper depend on the values listed in Table 1.

\begin{tabular}{|l|c|c|}
\hline Equipment & & Value \\
\hline Digital signal processing & $P_{\text {el/proc }}$ & $100 \mathrm{~W}$ \\
\hline Power amplifier (SISO) & $\eta$ & $12.8 \%$ \\
\cline { 2 - 3 } & $P_{\text {el/amp }}$ (max.) & $156 \mathrm{~W}$ \\
\hline Power amplifier (MIMO) & $\eta$ & $11.54 \%$ \\
\cline { 2 - 3 } & $P_{\text {el/amp }}$ (max.) & $10.4 \mathrm{~W}$ \\
\hline Transceiver & $P_{\text {el/trans }}$ & $100 \mathrm{~W}$ \\
\hline Rectifier & $P_{\text {el/rect }}$ & $100 \mathrm{~W}$ \\
\hline Air conditioning & $P_{\text {el/airco }}$ & $225 \mathrm{~W}$ \\
\hline Microwave link & $P_{\text {el/micro }}$ & $80 \mathrm{~W}$ \\
\hline
\end{tabular}

Table 1: Power consumption of the base station components for the considered technologies (mobile WiMAX, fixed WiMAX, UMTS, HSPA and LTE).

The most important source of power consumption is the air conditioning. In contrary to [16], the same air conditioning is used for all technologies. This adaptation is made based on the information retrieved from operators. Furthermore, a power amplifier with a more realistic efficiency was chosen for the reference configuration [13]. This power amplifier can be used for all the considered technologies because it supports the frequency of each considered technology and the RF output power of the power amplifier covers the needed input power of the antennas for each considered technology. Also the power amplifier for the extended configuration can be used for all the considered technologies.

As a validation of our model, we compare the power consumption with available data and measurements. For a 3-sector base station with one antenna per sector, $P_{e l}$ equal to $1672.6 \mathrm{~W}$ is found with 
eq. (3) for UMTS, HSPA and LTE. In [13] and [15], $P_{e l}$ of $1700 \mathrm{~W}$ and $1500 \mathrm{~W}$, respectively, are found for the traditional $3 \mathrm{G}$ base station which is similar to the $P_{e l}$ obtained with our model. In [4], $P_{e l}$ for a 1-sector base station with one antenna is $783 \mathrm{~W}$. With our model, similarly, $P_{e l}=761 \mathrm{~W}$, is obtained. Furthermore, a good similarity between our $P_{e l}$ and confidential data from an operator about the power consumption of $3 \mathrm{G}$ base stations is obtained.

\subsection{Calculation of the coverage range $R$ of the base station}

The power consumption $P_{e l}$ of the base station is now related to the wireless range $R$ covered by this base station. To this end, a link budget has to be constructed. A link budget takes all of the gains and the losses of the transmitter through the medium to the receiver into account. Firstly, we calculate the maximum allowable path loss $P L_{\max }$ (in $\mathrm{dB}$ ) to which a transmitted signal can be subjected while still being detectable at the receiver. The path loss is the ratio of the radiated power to the received power of the signal, it includes all of the possible elements of loss associated with interactions between the propagating wave and any objects between the transmit and receive antennas [29]. To determine $P L_{\max }$, the parameters of Table 2 are taken into account. Table 2 lists all the gains and losses that occur. These parameters are retrieved from the specifications and/or are typical values proposed by the operators themselves in order to make a fair comparison between the considered technologies.

\begin{tabular}{|c|c|c|c|c|c|c|}
\hline Parameter & Mobile WiMAX & Fixed WiMAX & UMTS & HSPA & LTE & Unit \\
\hline Frequency & 2.5 & 3.5 & 2.1 & 2.1 & 2.6 & $\mathrm{GHz}$ \\
\hline Input power of base station $P_{T x}$ & 35 & 35 & 43 & 43 & 43 & $\mathrm{dBm}$ \\
\hline Effective input power of base station $P_{T x}^{T C H}$ & 35 & 35 & 31.5 & 24.7 & 43 & $\mathrm{dBm}$ \\
\hline Antenna gain of base station & 16 & 17 & 17.4 & 17.4 & 18 & $\mathrm{dBi}$ \\
\hline Antenna gain of mobile station & 2 & 8 & 0 & 0 & 0 & $\mathrm{dBi}$ \\
\hline Number of MIMO Tx antennas & $1,2,3,4$ & 1 & 1 & $1,2,3,4$ & $1,2,3,4$ & - \\
\hline Number of MIMO Rx antennas & $1,2,3,4$ & 1 & 1 & $1,2,3,4$ & $1,2,3,4$ & - \\
\hline Cyclic combining gain of base station & 3 & 3 & 3 & 3 & 3 & $\mathrm{~dB}$ \\
\hline Soft handover gain & 0 & 0 & 1.5 & 1.5 & 0 & $\mathrm{~dB}$ \\
\hline Feeder loss of base station & 0.5 & 0.5 & 2 & 0 & 2 & $\mathrm{~dB}$ \\
\hline Feeder loss of mobile station & 0 & 0 & 0 & 0 & 0 & $\mathrm{~dB}$ \\
\hline Fade margin & 10 & 10 & 10 & 10 & 10 & $\mathrm{~dB}$ \\
\hline Yearly availability & 99.995 & 99.995 & 99.995 & 99.995 & 99.995 & $\%$ \\
\hline Cell interference margin & 2 & 0 & 0 & 2 & 2 & $\mathrm{~dB}$ \\
\hline User interference margin & 0 & 0 & 6 & 9 & 0 & $\mathrm{~dB}$ \\
\hline Bandwidth & 1.25 & 3.5 & 5 & 5 & 1.4 & $\mathrm{MHz}$ \\
\hline Constellation & $2 / 364-\mathrm{QAM}$ & 3/4 QPSK & PS 384 data service & $3 / 4$ QPSK & $\begin{array}{l}{[2 / 3 \text { 16-QAM, }} \\
2 / 3 \text { 64-QAM }]\end{array}$ & - \\
\hline Receiver SNR & 19 & 11.2 & 7 & 3.4 & {$[19,29.4]([30])$} & $\mathrm{dB}$ \\
\hline Number of used subcarriers & 85 & 201 & 1 & 1 & 76 & - \\
\hline Number of total subcarriers & 128 & 256 & 1 & 1 & 128 & - \\
\hline Noise figure of mobile station & 7 & 4.6 & 8 & 9 & 8 & $\mathrm{~dB}$ \\
\hline Implementation loss of mobile station & 2 & 0 & 0 & 0 & 0 & $\mathrm{~dB}$ \\
\hline Processing gain & - & - & 10.0 & 12 & - & $\mathrm{dB}$ \\
\hline Control overhead & - & - & 0.25 & 0.25 & - & - \\
\hline Target load & - & - & 0.75 & 0.875 & - & - \\
\hline Max. number of users & - & - & 4 & 75 & - & - \\
\hline Duplexing & \multicolumn{5}{|c|}{ TDD (Time Division Duplexing) } & - \\
\hline Building penetration loss [31] & 8.1 & 8.1 & 8.1 & 8.1 & 8.1 & $\mathrm{~dB}$ \\
\hline
\end{tabular}

Table 2: Link budget table for considered technologies.

Some of these parameters need a short explanation like e.g. the fading margin. The fading margin 
accounts for temporal fading (e.g., varying weather conditions) and is determined based on the projected yearly availability of the system. The noise figure is a measure of degradation of the SNR (Signal-toNoise Ratio) caused by components in the radio frequency signal chain. The receiver SNR determines the required SNR at the receiver for a certain BER (Bit Error Rate) and the bit rate.

Because UMTS and HSPA use W-CDMA as multiple access technique, an extra gain needs to be taken into account. This gain is called the processing gain $P G($ in $\mathrm{dB})$ and is defined as [32]:

$$
P G=-10 \cdot \log (S P)=-10 \cdot \log \left(\frac{C R}{S R}\right)
$$

with $S P$ the spreading factor which is the ratio of the chip rate $C R$ (in Mcps) to the symbol rate $S R$ (in bps). The processing gain is thus the ratio of the spreaded (RF) bandwidth to the unspreaded (baseband) bandwidth. Also the input power of the antenna for UMTS and HSPA needs to be scaled according to the control overhead, the target load, and the maximum number of users [33]:

$$
P_{T x}^{T C H}=\frac{(1-C L) \cdot P_{T x}}{T L \cdot N_{u s e r s}}
$$

with $P_{T x}^{T C H}$ the power reserved by the base station for the traffic channels. $C L$ is the control overhead, $T L$ the target load and $N_{\text {users }}$ the maximum number of users. $P_{T x}$ is used in order to determine the power consumption of the base station and $P_{T x}^{T C H}$ is used to determine the range of the UMTS and HSPA base station (Table 2). For mobile WiMAX, fixed WiMAX, and LTE, $P_{T x}$ in Table 2 is equal to $P_{T x}^{T C H}$ because an OFDMA based multiple access technology is used. Also, the user interference margin UIM (in $\mathrm{dB}$ ) needs to be taken into account when using UMTS and HSPA [33]:

$$
U I M=-10 \cdot \log _{10}(1-T L)
$$

with $T L$ the target load.

For mobile WiMAX, HSPA and LTE an extra gain, the MIMO gain $G_{M I M O}$, needs to be taken into account for the extended configuration (MIMO) (Section 4.3). Here, the theoretical MIMO gain $G_{M I M O}$ is considered [34]:

$$
G_{M I M O}=10 \cdot \log _{10}\left(n_{T x} \cdot n_{R x}\right)
$$

$G_{M I M O}$ in eq. (7) might be an overestimation for some realistic cases [35], but eq. (7) is used for all technologies in order to have a fair comparison.

Once the maximum allowable path loss $P L_{\max }$ is known, the maximum range $R$ (in metres) covered 
by the base station of a certain technology can be determined:

$$
R=g^{-1}\left(\left(P L_{\max }-S M\right) \mid f, h_{B S}, h_{M S}\right)
$$

with $P L_{\max }$ the maximum allowable path loss (in $\mathrm{dB}$ ), $S M$ the shadowing margin (in $\mathrm{dB}$ ), $f$ the frequency (in $\mathrm{Hz}$ ),$h_{B S}$ the height of the base station (in metres) and $h_{M S}$ the height of the mobile station (in metres). The shadowing margin depends on the standard deviation of the path loss model, the coverage percentage and the outdoor standard deviation. Here, a coverage percentage of $90 \%$ is considered. The function $g($.$) depends on the used path loss model e.g., the HATA model and the Erceg model [36,37]$. In this paper, the Erceg $\mathrm{C}$ model is used as this is best suitable for suburban areas. The quantity before the "|" in eq. (8) is a variable and varies over a continuous interval, while the quantities after the "|" are parameters which take only one discrete known value.

\subsection{Parameter to quantify the power consumption and efficiency}

If multiple technologies are compared, it is very difficult to determine which one is the most energyefficient: one technology could have higher power consumption but also a higher range, another one could have a smaller range but also a lower power consumption etc. Therefore, the power consumption $P C_{\text {area }}$ per covered area (in $\mathrm{W} / \mathrm{m}^{2}$ ) is defined to quantify the power consumption and efficiency for different technologies:

$$
P C_{\text {area }}=\frac{P_{e l}}{\pi \cdot R^{2}}
$$

with $P_{e l}$ the power consumption of the entire base station (in Watt) and $R$ the covered range (in $\mathrm{m}$ ). This parameter allows us to compare the energy efficiency of different wireless technologies and to determine which one is the most energy-efficient. The lower $P C_{\text {area }}$, the more energy-efficient the considered technology is. The normalization to the area allows us to make a fair comparison between the different technologies in terms of energy efficiency. It is assumed that the cells are circular.

\section{Applications}

\subsection{Configuration}

In this investigation, the base stations are placed outdoor in a suburban environment. Only macro cells with a base station antenna height of $30 \mathrm{~m}$ are considered. For the mobile stations, an indoor residential configuration with a WNIC (Wireless Network Interface Card) for a laptop for all technologies is considered except for fixed WiMAX, where we consider a residential gateway. Table 3 summarizes the 
configuration parameters for all technologies described in Section 2.

\begin{tabular}{|l|c|}
\hline Parameter & Value \\
\hline Area type & suburban \\
\hline Number of sectors $n_{\text {sector }}$ & 3 \\
\hline Height of a base station & $30 \mathrm{~m}$ \\
\hline Height of a mobile station & $1.5 \mathrm{~m}$ \\
\hline Coverage requirement & $90 \%$ \\
\hline Path loss model & Erceg C \\
\hline Shadowing margin & $13.2 \mathrm{~dB}$ \\
\hline
\end{tabular}

Table 3: Configuration table under consideration.

We also define two technical configurations for the outdoor base stations: a basic reference configuration and an extended configuration. All the considered technologies support the basic reference configuration. The extended configuration is only supported by mobile WiMAX, HSPA and LTE. In the basic reference configuration, one transmitting $(\mathrm{Tx})$ and one receiving $(\mathrm{Rx})$ antenna is considered, i.e., a SISO system. In the extended configuration, both the base station and the receiver have multiple antennas. Six different MIMO (Multiple Input Multiple Output) systems are considered: 2x1 (2 Tx and $2 \mathrm{Rx}), 2 \times 2,2 \times 3,3 \times 3,4 \times 3$ and 4x4 MIMO systems.

The frequencies used for the link budget calculations of the different technologies are the following: 2.5 GHz for mobile WiMAX, $3.5 \mathrm{GHz}$ for fixed WiMAX, $2.1 \mathrm{GHz}$ for UMTS and HSPA, and $2.6 \mathrm{GHz}$ for LTE.

\subsection{Comparison of the considered technologies}

In this section, the considered wireless technologies are compared for the reference configuration. In order to make a fair comparison, predefined bit rates of $3 \mathrm{Mbps}$ and $60 \mathrm{Mbps}$ are considered. Only mobile WiMAX and LTE support 60 Mbps. The different parameters can be found in Tables 1, 2 and 3, . For $60 \mathrm{Mbps}$, a $20 \mathrm{MHz}$ channel is used. Mobile WiMAX uses 1440 out of 2048 subcarriers and LTE 1201. Furthermore, the 2/3 64-QAM modulation (19 dB receiver SNR for mobile WiMAX and $29.4 \mathrm{~dB}$ for LTE [30]) is used.

Table 4 lists the results for $R, P_{e l}$ and $P C_{\text {area }}$. Based on the assumptions made for the parameters and $3 \mathrm{Mbps}$, UMTS is the most energy-efficient technology (lowest $P C_{\text {area }}$ ) followed by (in rising order for $P C_{\text {area }}$ ) fixed WiMAX, LTE, mobile WiMAX and HSPA.

The power efficiency $P C_{\text {area }}$ of UMTS and fixed WiMAX is considerably lower $\left(<1 \mathrm{~mW} / \mathrm{m}^{2}\right)$ than those for mobile WiMAX, HSPA, and LTE $\left(2.4-3.5 \mathrm{~mW} / \mathrm{m}^{2}\right)$. UMTS performs better than fixed WiMAX because of its higher ranges (lower receiver SNR and the processing gain in Table 2). The higher power consumption $P_{e l}$ of UMTS is due to the higher input power $P_{T x}$ of the antenna. Fixed WiMAX is more 


\begin{tabular}{|l|c|c|c|c|c|}
\hline 3 Mbps & Mobile WiMAX & Fixed WiMAX & UMTS & HSPA & LTE \\
\hline Bit rate $[\mathrm{Mbps}]$ & 3.6 & 3.1 & 3 & 3.8 & 3.2 \\
\hline$R[\mathrm{~m}]$ & 342.5 & 674.4 & 846.1 & 372.5 & 470.6 \\
\hline$P_{e l}[\mathrm{~W}]$ & 1279.1 & 1279.1 & 1672.6 & 1672.6 & 1672.6 \\
\hline$P C_{\text {area }}\left[\mathrm{mW} / \mathrm{m}^{2}\right]$ & 3.5 & 0.9 & 0.7 & 3.8 & 2.4 \\
\hline \hline $\mathbf{6 0}$ Mbps & Mobile WiMAX & Fixed WiMAX & UMTS & HSPA & LTE \\
\hline Bit rate $[\mathrm{Mbps}]$ & 61.1 & - & - & - & 67.6 \\
\hline$R[\mathrm{~m}]$ & 172.2 & - & - & - & 138.3 \\
\hline$P_{\text {el }}[\mathrm{W}]$ & 1279.1 & - & - & - & 1672.6 \\
\hline$P C_{\text {area }}\left[\mathrm{mW} / \mathrm{m}^{2}\right]$ & 13.7 & - & - & - & 27.8 \\
\hline
\end{tabular}

Table 4: Comparison of the considered technologies for a physical bit rate of approximately $3 \mathrm{Mbps}$ and 60 Mbps.

efficient than mobile WiMAX, HSPA and LTE because of its higher range (lower receiver SNR and higher antenna gain of the mobile station in Table 2) and its lower power consumption (lower $P_{T x}$ of the fixed WiMAX base station). Finally, LTE is more energy efficient than mobile WiMAX and HSPA because of its higher effective input power $P_{T x}^{T C H}$ of the antenna resulting in a higher range.

For 60 Mbps, mobile WiMAX performs better than LTE due to its higher range and lower power consumption. This higher range is caused by its lower receiver SNR. The power consumption is lower because of its higher $P_{T x}$.

Important to remark is that for different modulation schemes and coding rates, the power consumption $P_{e l}$ does not change [16]. However, a different range is obtained which has a direct influence on $P C_{a r e a}$.

\subsection{Influence of MIMO}

In this section, the influence of MIMO on the energy efficiency is investigated. The considered technologies are compared for a $2 \times 1,2 \times 2,3 \times 2,3 \times 3,4 \times 3$ and $4 \times 4$ MIMO system (Section 4.1). Fig. 2 gives an overview of $P C_{\text {area }}$ as a function of the chosen MIMO system for mobile WiMAX, HSPA and LTE. The energy efficiency gain is also indicated in the figure. The energy efficiency gain $E G$ indicates how much (as a percentage) $P C_{\text {area }}$ has decreased compared to the SISO system:

$$
E G=\frac{P C_{\text {area } / S I S O}-P C_{\text {area } / M I M O}}{P C_{\text {area }} / \text { SISO }} \cdot 100
$$

Based on the assumptions made for the parameters and the considered cases, Fig. 2 shows that the energy efficiency increases when MIMO is introduced. The highest energy efficiency is obtained with a $4 \mathrm{x} 4$ MIMO system (up to $63 \%$ ). EG (Energy efficiency Gain) is the highest for mobile WiMAX.

Compared to the SISO system, the area covered by each technology increases with $438 \%$, due to an increase of $132 \%$ of the range, while the power consumption increases with only $95 \%$ for mobile WiMAX and $173 \%$ for HSPA and LTE. The increase in power consumption is lower for mobile WiMAX because 


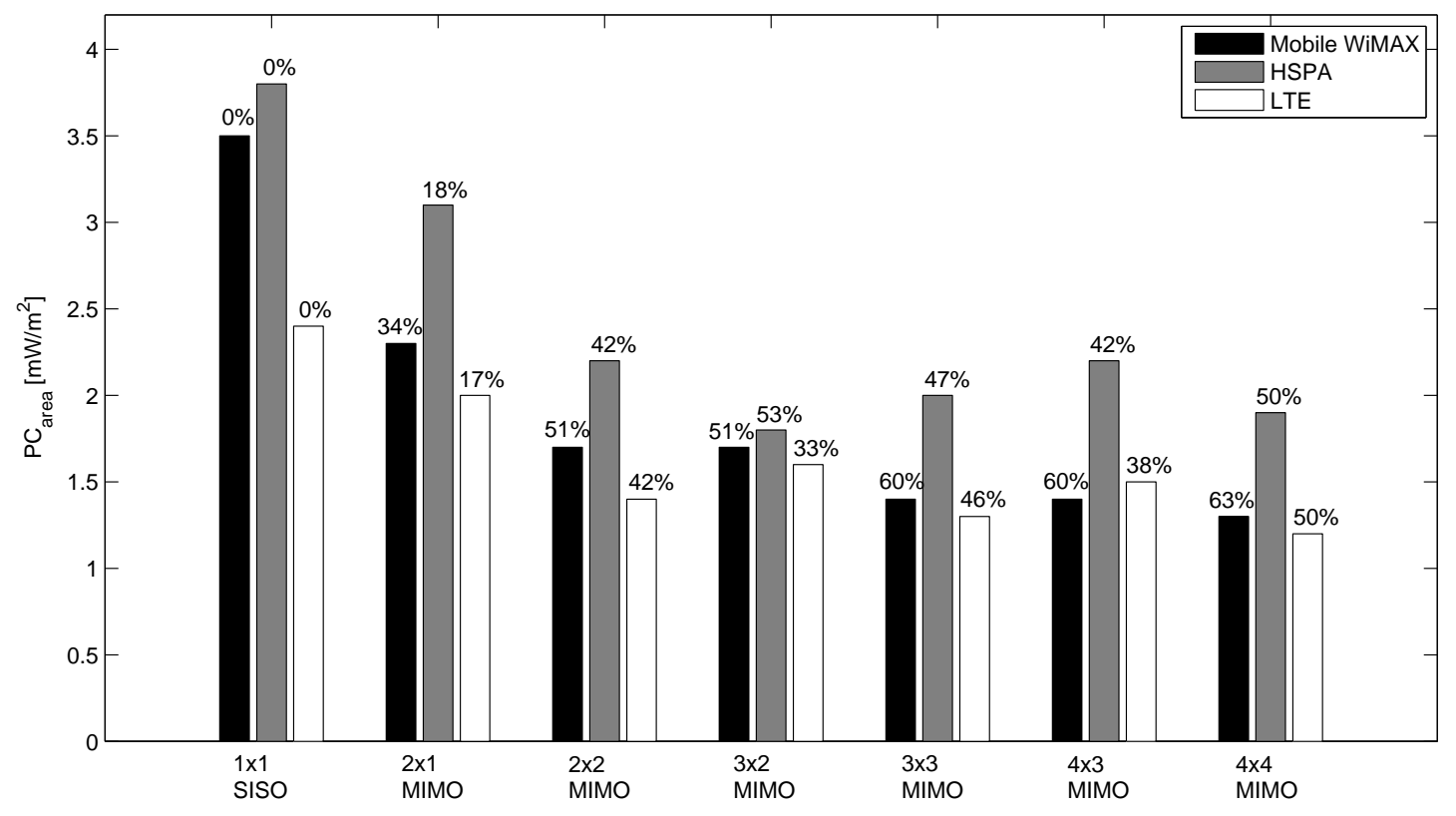

Figure 2: Influence of $2 \times 1,2 \times 2,3 \times 2,3 \times 3,4 \times 3$ and $4 \times 4$ MIMO on $P C_{\text {area }}$.

of its lower input power $P_{T x}$ of the antenna (Table 2). This is also the reason why the highest $E G$ are obtained with mobile WiMAX (34 \% - 63 \%) for all considered MIMO systems.

Comparing the different MIMO systems reveals that a higher MIMO array size (i.e., more transmitting and/or more receiving antennas) does not always results in a higher energy efficiency. For mobile WiMAX, $E G$ for a 2x2 and 3x2 MIMO system are approximately equal (51\%). This can be explained as follows. The power consumption $P_{e l}$ of the base station for $2 \times 2$ MIMO is lower $(1689.5 \mathrm{~W}$ versus $2495.8 \mathrm{~W}$ for $3 \times 2$ MIMO) because only 2 transmitting antennas are used (eq. (3)). However, the range is higher for the 3x2 MIMO system (794.4 m versus $576.3 \mathrm{~m}$ for 2x2 MIMO) because of its higher MIMO gain (eq. (7)), resulting in similar values for $P C_{\text {area }}$ and $E G$.

Analogously for LTE, the $2 \times 2$ and $3 \times 3$ MIMO system have higher $E G$ than the $3 \times 2$ and the $4 \times 3$ MIMO system, respectively. For HSPA even lower $E G$ values for the $3 \times 3$ and $4 \times 3$ MIMO system than for the 3x2 MIMO system and the 3x3 MIMO system are obtained, respectively.

\section{Conclusions and future research}

In this paper, the power consumption for five different wireless technologies, namely mobile WiMAX, fixed WiMAX, UMTS, HSPA and LTE is investigated based on the parameter assumptions for the five technologies. This power consumption is then related to the coverage of the base station. The base stations (macro cells) are placed outdoor and for the mobile stations an indoor residential scenario with 
a Wireless Network Interface Card (WNIC) is considered, except for fixed WiMAX where a residential gateway is considered. The energy efficiency per covered area $P C_{a r e a}$ was defined and compared for the considered bit rates for a basic reference configuration and an extended future configuration. Lower $P C_{\text {area }}$ values mean that the technology is more energy-efficient.

Based on the assumptions made for the parameters, the reference configuration, and 3 Mbps, UMTS is the most energy-efficient technology followed by (in rising order of $P C_{\text {area }}$ ) fixed WiMAX, LTE, mobile WiMAX and HSPA. For 60 Mbps (only supported by mobile WiMAX and LTE), mobile WiMAX performs better.

The introduction of MIMO has a positive influence on the energy efficiency. The biggest influence is obtained with a 4x4 MIMO system: $P C_{\text {area }}$ increases up to $63 \%$ for mobile WiMAX and up to $50 \%$ for HSPA and LTE. Furthermore, a higher MIMO size does not always result in a higher energy efficiency.

Future research will consist of including micro cells to cover smaller areas in the model of Section 3. Also the influence of load dependency on the range (cell breathing) and thus the power efficiency will be investigated. When there is little or no activity in the area of the base station, the base station could be switched off (sleep mode). Nowadays, this is not supported by the base station but this should be part of future research. The sleep modes have to be combined with an advanced management algorithm and will have a positive influence on the power consumption and energy efficiency.

\section{Acknowledgment}

W. Joseph is a Post-Doctoral Fellow of the FWO-V (Research Foundation Flanders).

\section{References}

[1] M. Pickavet, W. Vereecken, S. Demeyer, P. Audenaert, B. Vermeulen, C. Develder, D. Colle, B. Dhoedt, and P. Demeester, "Worldwide Energy Needs for ICT: the Rise of Power-Aware Networking," in 2008 IEEE ANTS Conference, Bombay, India, December 2008, pp. 1-3.

[2] Ericsson, "Sustainable energy use in mobile communications," White paper, August 2007.

[3] M. Etoh, T. Ohya, and Y. Nakayama, "Energy Consumption Issues on Mobile Network Systems," in International Symposium Issues on Mobile Network Systems, 2008, pp. 365-368.

[4] F. Richter, A. Fehske, and G. Fettweis, "Energy Efficiency Aspects of Base Station Deployment Strategies for Cellular Networks," in IEEE 70th Vehicular Technology Conference Fall (VTC 2009Fall), 2009, pp. $1-5$. 
[5] G. Koutitas, "Low Carbon Network Planning," in European Wireless Conference, 2010, Lucca, Italy, April, pp. $411-417$.

[6] G. Micallef, P. Mogensen, and H. Scheck, "Cell Size Breathing and Possibilities to Introduce Cell Sleep Mode," in European Wireless Conference, 2010, Lucca, Italy, April, pp. 111 - 115.

[7] Part 16: Air Interface for Fixed and Mobile Broadband Wireless Access Systems: Amendment 2: Physical and Medium Access Control Layers for Combined Fixed and Mobile Operation in Licensed bands and Corrigendum 1, IEEE Computer Society and the IEEE Microwave Theory and Techniques Society, February 2006. [Online]. Available: www.ieee802.org/16

[8] Air Interface for Fixed Broadband Wireless Access Systems, IEEE Computer Society and the IEEE Microwave Theory and Techniques Society, October 2004. [Online]. Available: www.ieee802.org/16

[9] 3rd Generation Partnership Project; Technical Specification Group Radio Access Network; UE Radio Transmission and Reception (TDD) (Release 1999), TR 25.102 v3.13.0, 3GPP, October 2006.

[10] 3rd Generation Partnership Project: Technical Specification Group Radio Access Network: Physical layer aspects of UTRA High Speed Downlink Packet Access (Release 4), TR 25.848 v4.0.0., 3GPP, March 2001.

[11] LTE: 3rd Generation Partnership Project: Technical Specification Group Radio Access Network: Evolved Universal Terrestrial Radio Access (E-UTRA): User Equipment (UE) radio transmission and reception (TS 36.101 v9.1.0 Release 9) , 3GPP, September 2009.

[12] TeliaSonera, "TeliaSonera first in the world with 4G services, www.teliasonera.com/News-andArchive/Press-releases/2009/TeliaSonera-first-in-the-world-with-4G-services/," December 2009.

[13] U. Barth, "Wireless Networks, EARTH research project," ETSI Green Agenda Workshop, November 2009.

[14] P. Gildert, "Power System Efficiency in Wireless Communication," Ericsson, January 2006.

[15] L. Hérault, "Green Wireless Communications eMobility GA1," e-Mobility Workshop at the 4th Future Networks Concertation Meetings, Brussels, Belgium, September 2009.

[16] M. Deruyck, W. Vereecken, E. Tanghe, W. Joseph, M. Pickavet, L. Martens, and P. Demeester, "Power consumption in wireless access networks," in European Wireless Conference, 2010, Lucca, Italy, April 2010, pp. $924-931$. 
[17] — - "Comparison of power consumption of mobile WiMAX, HSPA and LTE access networks," in 9th Conference of Telecommunication, Media and Internet Techno-Economics (CTTE), 2010, Ghent, Belgium, June 2010, paper ID: 17.

[18] Pfannenberg, "http://www.pfannenbergusa.com/catalog/catalog-downloads/2010_Catalog.pdf," 2010.

[19] F. Raab, P. Asbeck, S. Cripps, P. Kenington, Z. Popovic, N. Pothecary, J. Sevic, and N. Sokal, "RF and Microwave Power Amplifiers and Transmitter Technologies - Part 1," High Frequency Electronics, pp. 22 - 36, May 2003.

[20] R. Bhagavatula, R. H. Jr., and K. Linehan, "Performance Evaluation of MIMO Base Station Antenna Designs," Antenna Systems $\&$ Technology, vol. 11, no. 6, pp. 14 - 17, November - December 2008.

[21] Ophir RF model 5303009, 2010.

[22] Ophir RF model 5303025, 2010.

[23] Ophir RF model 5303075, 2010.

[24] Power-One S series, 2010.

[25] Daikin FUQ125BW13/R2Q125D7413, 2010.

[26] Allgon Microwave - AMR Transcend PLUS, 2010.

[27] Ceragon Networks - FibeAIR 1500P Family, 2010.

[28] Trangobroadbandnetworks - TrangoLINK - Giga, 2010.

[29] S. Saunders, Antennas and Propagation for Wireless Communication Systems. Wiley, 1999.

[30] S. Abdul Basit, "Dimensioning of the LTE network: Description of Models and Tools, Coverage and Capacity Estimation of 3GPP Long Term Evolution radio interface," Master's thesis, Helsinki Unversity of Technology, February 2009.

[31] D. Plets, W. Joseph, L. Verloock, L. Martens, H. Gauderis, and E. Deventer, "Extensive Penetration Loss Measurements and Models for Different Building Types for DVB-H in the UHF Band," IEEE Transactions on broadcasting, vol. 55, no. 2, pp. 213 - 222, June 2009.

[32] R. Krüger and H. Mellein, UMTS Introduction and Measurement. Rohde \& Schwarz, 2004. 
[33] J. Hämäläinen, "Cellular Network Planning and Optimization - Part VII: WCDMA link budget," Helsinki University of Technology, Course, 2008. [Online]. Available: http://www.comlab.hut.fi/studies/3275/Cellular_network_planning_and_optimization_part8.pdf

[34] L. Nuaymi, WiMAX Technology for Broadband Wireless Access. Wiley, 2007.

[35] M. Deruyck, E. Tanghe, W. Joseph, D. Pareit, I. Moerman, and L. Martens, "Performance Analysis of WiMAX for mobile Applications," in IEEE Wireless Communications and Networking Conference, Sydney, Australia, April 2010, paper ID: T04S35P01.

[36] M. Hata, "Empirical Formula for Propagation Loss in Land Mobile Radio Services," IEEE Transactions on Vehicular Technology, vol. 29, no. 3, pp. 317 - 325, August 1980.

[37] V. Erceg, L. Greenstein, S. Tjandra, S. Parkoff, A. Gupta, B. Kulic, A. Julius, and R. Bianchi, "An Empirically Based Path Loss Model for Wireless Channels in Suburban Environments," IEEE Journal on Selected Areas in Communications, vol. 7, no. 7, pp. 1205 - 1211, July 1999. 